\title{
A study on the cleanser for terrestrial heating pipe
}

\author{
Zhao Di ${ }^{1, a}$, Zhao Hai ${ }^{1, b, *}$, Liu Junqing ${ }^{2, c}$, Hu Yixiang ${ }^{1, d}$ and Wang Hao ${ }^{1, e}$ \\ ${ }^{1}$ New Resources Department, Shenyang Institute of Engineering, Daoyi Street, Shenyang, China \\ ${ }^{2}$ Automation Department, Shenyang Institute of Engineering, Daoyi Street, Shenyang, China \\ a email:junqing_liu@126.com, b email:hai365@126.com, cemail:hai19710225@126.com \\ *corresponding author: Zhao Hai
}

Keywords: chemical cleanser, terrestrial heating pipe.

\begin{abstract}
In this paper, the cleanser for was prepared using sulfouren, citric acid and sulfamic acid. The performance of the chemical cleanser on corrosion and corrosion inhibition was studied. The results showed that, the rust deposit ratio of the cleanser at the regulated cleaning condition exceeds 95\%, and the cleaner showed a good corrosion inhibition performance. The biodegradation rate exceeded $95 \%$.
\end{abstract}

\section{Introduction}

Terrestrial heating pipe is widely used in the northern of China. After a long time of usage, the inner part of the pipe would yield a large amount of slime, bacteria and scale. Pipe lining, forming pipe blockage, resulting in poor circulation, insufficient heat dissipation would affect heating temperature and heating effect. The data show that the earth warms after a year of usage, every increase $1 \mathrm{~mm}$ of dirt, the wall causes a $6{ }^{\circ} \mathrm{C}$ decline in heating indoor temperature. This not only affects the normal use of the heating pipe, but also causes the waste of energy. Such a long time without effective cleaning treatment will cause the geothermal heating system to fail, thus cannot be used in the pipe, or even destroy the ground, dismantle or replace the geothermal energy. Piping system, to the floor heating users caused property loss and life inconvenience. There are three kinds of methods on terrestrial heating pipe cleaning, include ejection cleaning, pulse cleaning, and chemical cleaning. When the high-pressured gas is driven by the high speed in the terrestrial heating pipe, the front of the projectile move in a pipe with a hard solid can cause a lot of dirt. Solids do damage to geothermal pipes. At present, pulse cleaning is widely used for the application. The working principle is to wash the dirt with the pulse shock of air and remove the dirt from water and clean it. Chemical cleaning is the most thorough cleaning of all cleaning methods, because chemical agents dissolve the scale and rust of the water. In this study, the chemical cleaning method was used mainly include the amino sulfonic acid, citric acid. Dimethylphenylthiourea and sodium nitrite, the acid that is used will not corrode the geothermal pipeline and the metal part. In addition, the addition of additives also includes scale and rust prevention. It is of great significance for the protection of terrestrial heating pipe.

\section{Experimental Preparation}

The amino sulfonic acid, citric acid, o-xylene thiourea and sodium nitrite are put into the cone mixing machine with a certain proportion, and the finished product is mixed evenly. Corrosion rate of the determination is made by a certain concentration of detergent solution placed in the conical flask, the specimens of different material separately hanging in the conical flask of aqueous solution containing detergent, static test of $12 \mathrm{~h}$. The biodegradation performance was determined by the method of biological shaking bed. 


\section{Experimental results and discussion.}

3.1 Effect of the concentration of cleanser on the rust deposit rate

The experiment studied the influence of different concentration on the rust deposit rate at temperature of $30{ }^{\circ} \mathrm{C}$ for the time $30 \mathrm{~min}$.

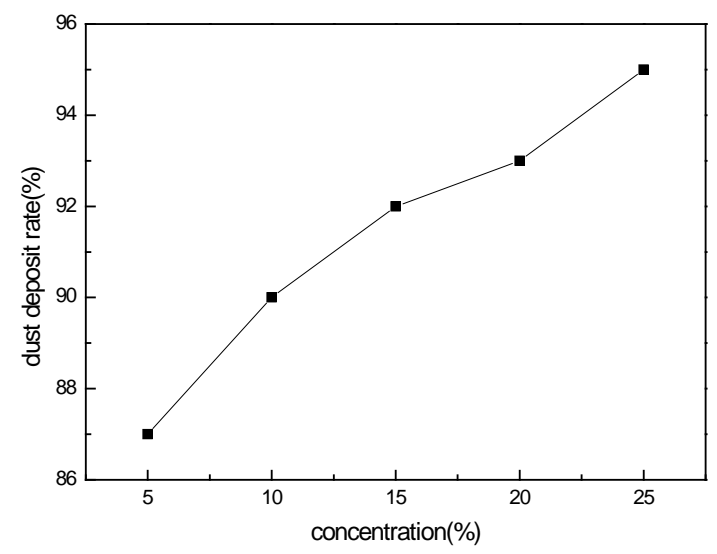

Figure 1 Influence of different concentration on the rust deposit rate

From the figure 1, it is can be seen that the rust deposit rate increased with the concentration of cleanser. When the concentration of cleanser was higher than $10 \%$, the removal rate was higher than $90 \%$. In the cleanser agent, amino sulfonic acid hydrolysis produce ammonium sulfate acid, can react with metal oxides in the heating pipe, generate amino sulfonic acid salt and other dissolved substances, and lower corrosive to metal. Therefore, as the main raw material of floor warm cleanser agent is appropriate. Considered the cleaning effect and the cost of agent, $15 \%$ of this cleanser agent is suitable to be used.

3.2 Effect of the temperature of cleanser on the rust deposit rate

The experiment studied the influence of different temperature of cleanser on the rust deposit rate at temperature of $20 \sim 50{ }^{\circ} \mathrm{C}$ for concentration of $15 \%$, the cleaning time for $30 \mathrm{~min}$.

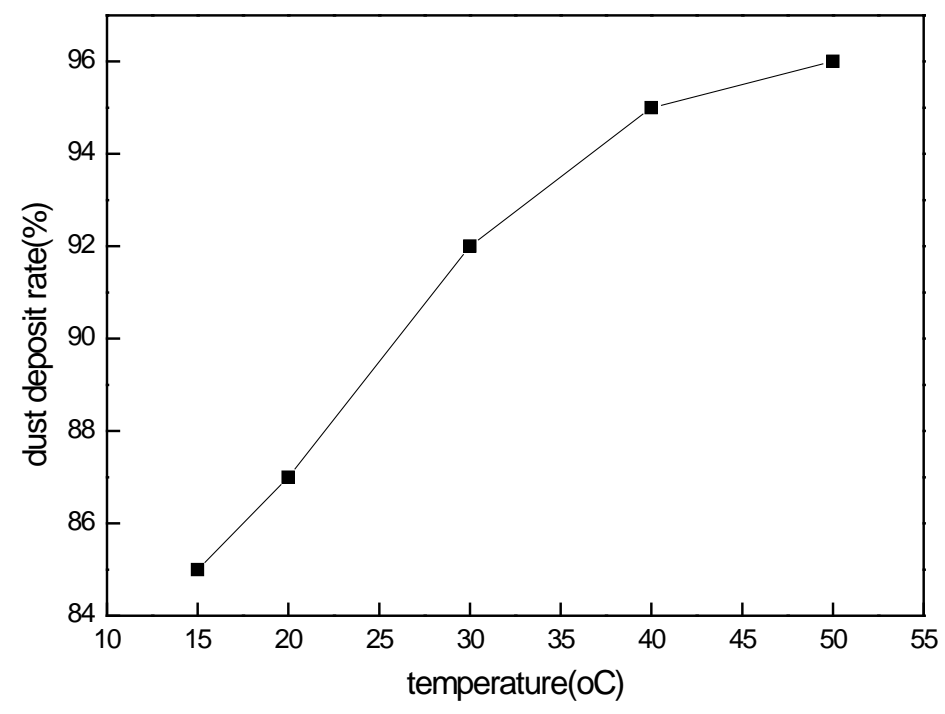

Figure 2 Influence of different temperature of cleanser on the rust deposit rate

From the figure 2, it is can be seen that the rust deposit rate increased with the temperature increases. The cleanser adding citric acid, citric acid small advantage is corrosion, non-toxic, high 
security. It can generate and iron ion complexing iron complex ions but need to adjust the solution $\mathrm{pH}$ value to 3.5 to 4 . Because the reaction rate of citric acid and iron ion is slow, the calcium and magnesium scale dissolving ability is slightly worse, it needs a certain high temperature when it be used. The increase of temperature can improve the rust deposit rate effectively. However, in the process of using citric acid, appropriate corrosion inhibited, and corrosion inhibitor should be added to reduce the corrosion of the acid to the metal. In addition, the high temperature can accelerate the corrosion of acid to metal. Thus, temperature of $30{ }^{\circ} \mathrm{C}$ is used in this research.

3.3. Effect of cleaning time on the rust deposit rate

The experiment studied the influence of cleaning time on the rust deposit rate at the temperature of $30{ }^{\circ} \mathrm{C}$ and the cleanser concentration is $15 \%$.

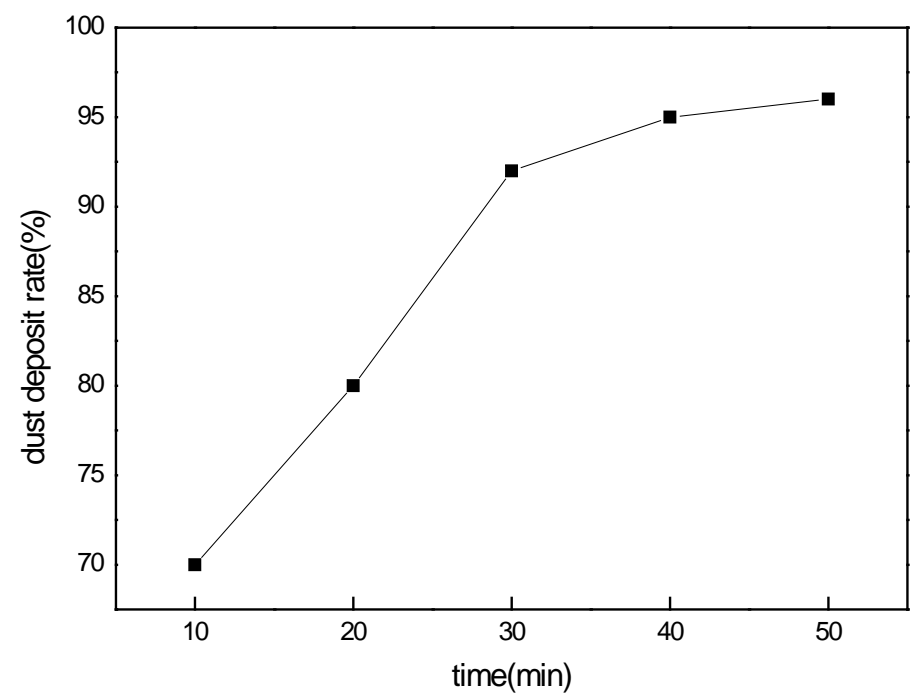

Figure 3 Influence of cleaning time on the rust deposit rate

From the figure 3, it is can be seen that the rust deposit rate is over $90 \%$ after $30 \mathrm{~min}$, and the rust deposit rate can reach $98 \%$ when the cleaning time is $50 \mathrm{~min}$. However, excessive time will affect working efficiency. The cleaning time used in this study is $30 \mathrm{~min}$.

3.4. Biodegradability of cleanser

The experiment studied the biodegradability of the cleanser. When determining the biodegradability of the cleaning agent, the concentration of the detergent was $50 \mathrm{mg} / \mathrm{L}$.

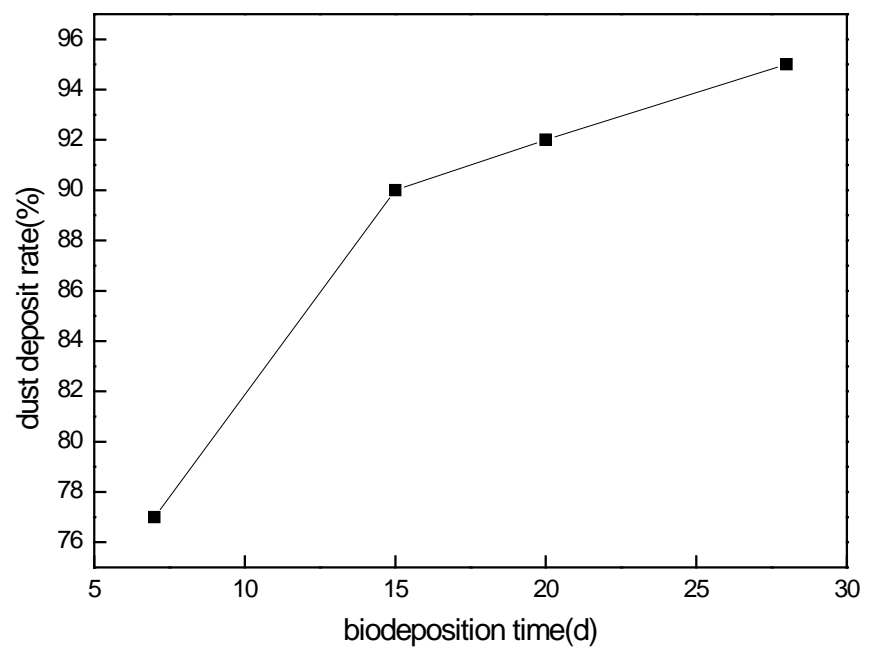

Figure 4 Biodegradability of the cleanser 
As seen from the figure 4, the biodegradation rate of cleaner is increasing with the increase of time. At the seventh day, the degradation rate has reached $78 \%$, the degradation rate of more than $90 \%$ after 14 days, as it reached to $95 \%$ to 28 days. Tt is shown that the cleanser is an easy degradation, safety and environmental friendly products.

\section{Conclusion}

(1) The cleanser prepared with amino sulfonic acid, citric acid, o-xylene thiourea has a good rust deposit performance at the condition of $30{ }^{\circ} \mathrm{C}$, the time $30 \mathrm{~min}$, and the concentration $15 \%$. The rust deposit rate can reach $95 \%$.

(2) The cleanser has low toxicity, friendly environment and good biodegradability.

\section{References}

[1] Zhang nanze, Liu Hailan, Han Sunyu. (2006) A study on the compounding recipe of nomal temperature solid cleanser for terrestrial heating pipe. Journel of Yanbian uniersity, 2, 114-117.

[2] Chen Jian-giu, Zeng Giu-mei, Shan Zhuo-ran, Yu Zhi-gang. (2003) Preparation of new-type special normal-temperature-used water-solubility metal washing agent for washing machine. surface technology.1, 41-45.

[3] Wang Wenbo, Zhao Hai, Liu Junqing. (2017) Research of cleanser for terrestrial pipe. Shandong chemical industry, 5, 39-40.

[4] Li Hongmei. (2008) Study and application of amidousulfoacid cleaning technology condition. Surface Technology, 3, 55-58.

[5] Li Xing. (2011) Boiler chemical cleaning methods of quantitative evaluation. Cleaning World, 7, 34-37. 